\title{
LEXSYG SMART - A LUMINESCENCE DETECTION SYSTEM FOR DOSIMETRY, MATERIAL RESEARCH AND DATING APPLICATION
}

\author{
DANIEL RICHTER ${ }^{1,2,3}$, ANDREAS RICHTER ${ }^{1}$ and KAY DORNICH ${ }^{1}$ \\ ${ }^{1}$ Freiberg Instruments GmbH, Delfter Str. 6, 09599 Freiberg, Germany \\ ${ }^{2}$ Leuphana University Lüneburg, Institute of Ecology, Subject Area Landscape Change, \\ Scharnhorststr. 1, C13.115, 21335 Lüneburg, Germany \\ ${ }^{3}$ Department of Human Evolution, MPI for Evolutionary Anthropology, Deutscher Platz 6, 04103 Leipzig, Germany
}

Received 2 October 2015

Accepted 24 November 2015

\begin{abstract}
Following the luminescence system lexsyg research, which was designed for research, the luminescence reader lexsyg smart for the application of luminescence detection was developed by Freiberg Instruments. It is suited for routine measurements of luminescence (thermoluminescence, photoluminescence, photon-stimulated, optically stimulated and infrared stimulated luminescence) for a wide range of materials because of the availability of several stimulation sources. The possibility for user definition and change of most parameters provides a great deal of flexibility and also allows research applications. While detection is limited to a single unit and sample storage to 40 positions, the lexsyg smart is much faster in aliquot transportation compared to the lexsyg research, and allows fast mass measurements in luminescence dating, retrospective and personal dosimetry, etc. Cross talk of optical stimulation is absent and cross-irradiation is negligible from the single radioactive source ( $\alpha, \beta$ or $\mathrm{x}$-ray) because of a disconnected sample storage wheel from the measurement chamber, which has a small volume and therefore gas consumption is small. Thermoluminescence measurements and pre-heatings are possible with a versatile heater, which can be programmed for linear/nonlinear heating at varying rates and durations for an almost unlimited number of steps. Optical excitation for up to three wavelength bands (violet, blue, green, yellow, infrared) is provided from high power LEDs or laser diodes, with an optional filter wheel to vary detection wavelength bands according the material specific requirements. Either can be programmed to change at almost any time within measurement sequences.
\end{abstract}

Keywords: luminescence equipment, dosimetry, luminescence dating, PL, TL-OSL, PSL.

\section{INTRODUCTION}

Luminescence detection in dosimetric application, especially in dating application, often requires the fast and efficient measurement of large numbers of sub-samples (aliquots), or the number of dosemeters to be measured in environmental/personal dosimetry is high. However, such

Corresponding author: D. Richter

e-mail: daniel.richter@freiberginstruments.com applications often require some degree of flexibility because of the use of a variety of dosimetric materials with different properties, requiring different parameters and measurement setups, like detection wavelength. Additionally, various ways to stimulate the luminescence for thermoluminescence (TL), photoluminescence (PL) photon-stimulated (PSL), optically stimulated (OSL), infrared stimulated luminescence (IRSL) measurements are required. While some luminescence detection systems are aimed towards research (e.g. Lapp et al., 2015; Richter et al., 2013), and thus being of rather large dimensions, others (e.g. Bortolot, 2000; Bortolot and Carriveau, 1982; 
Bøtter-Jensen and Bundgaard, 1978; Lapp et al., 2012; Mejdahl, 1982, Guérin and Lefèvre, 2014) provide a mix of equipment capable of research while being able to perform mass measurements. We here present a new development, lexsyg smart, by Freiberg Instruments, which combines some of the research capabilities and conceptional advantages of the lexsyg research (Richter et al., 2013; Lomax et al., 2014) in a cheaper, more compact as well as faster equipment.

\section{APPLICATION OF LUMINESCENCE DETEC- TION}

Luminescence of minerals and other phosphors is often used in dosimetry or dosimetric dating to determine the ionizing radiation dose some material, whether natural and artificial, has been exposed to. The fundamentals and basics of luminescence research have been summarized elsewhere, e.g. in Aitken (1985; 1998), BøtterJensen et al. (2003), McKeever (1985), Yukihara and McKeever (2011). Two major groups appear to be most important in application: One group includes among others radiation control, personal-, environmental-, fooddosimetry, as well as material research, while the other is mainly dosimetric dating application. The former requires the measurement of a large number of individual dosemeters and the latter often employs repeated measurement of many subsamples. Especially in luminescence dating many sub-samples (aliquots) are measured many times in a cyclic way, e.g. the single aliquot regeneration (SAR) protocol, in order to determine a single dose value. This is achieved through automated luminescence readers which have to be fast and efficient in order to process a large number of samples as quickly as possible. Apart from the target material specifications for measurement parameters, the limiting factors are the number of available positions for automated measurements, transport times and, in the case of optically stimulated luminescence (OSL), stimulation power.

In general, the most efficient equipment is optimized for a single task at the expense that it is usually not possible to deviate from fixed measurement parameters, preventing the measurement of e.g. other materials, or performing some research.

The lexsyg smart is aimed towards routine measurements research in dosimetry, material properties and dosimetric dating, while providing the possibility to study more than a single material and adapt the measurement parameters to a large extend, thus enabling research possibilities. The need of parameter adaptation is evident from the luminescence properties of, e.g. natural minerals (Fig. 1), which exhibit luminescence at different wavelengths. Because these different emissions have different properties, it is required to use specific excitation sources as well as being able to restrict detection wavelength by e.g. optical filters. Only equipment with the capacity to provide such flexibility allows the possibility to measure different materials within a single reader.

\section{THE LEXSYG SMART SYSTEM}

The lexsyg smart is a flexible luminescence reader (Fig. 2) for the measurement of thermally or optically stimulated luminescence (i.e. TL or OSL/IRSL/PL/PSL). It is designed for mass measurement capabilities, while providing the possibility to adapt and change fundamental measurement parameters to allow the measurement of a large variety of materials as well as opening research possibilities.

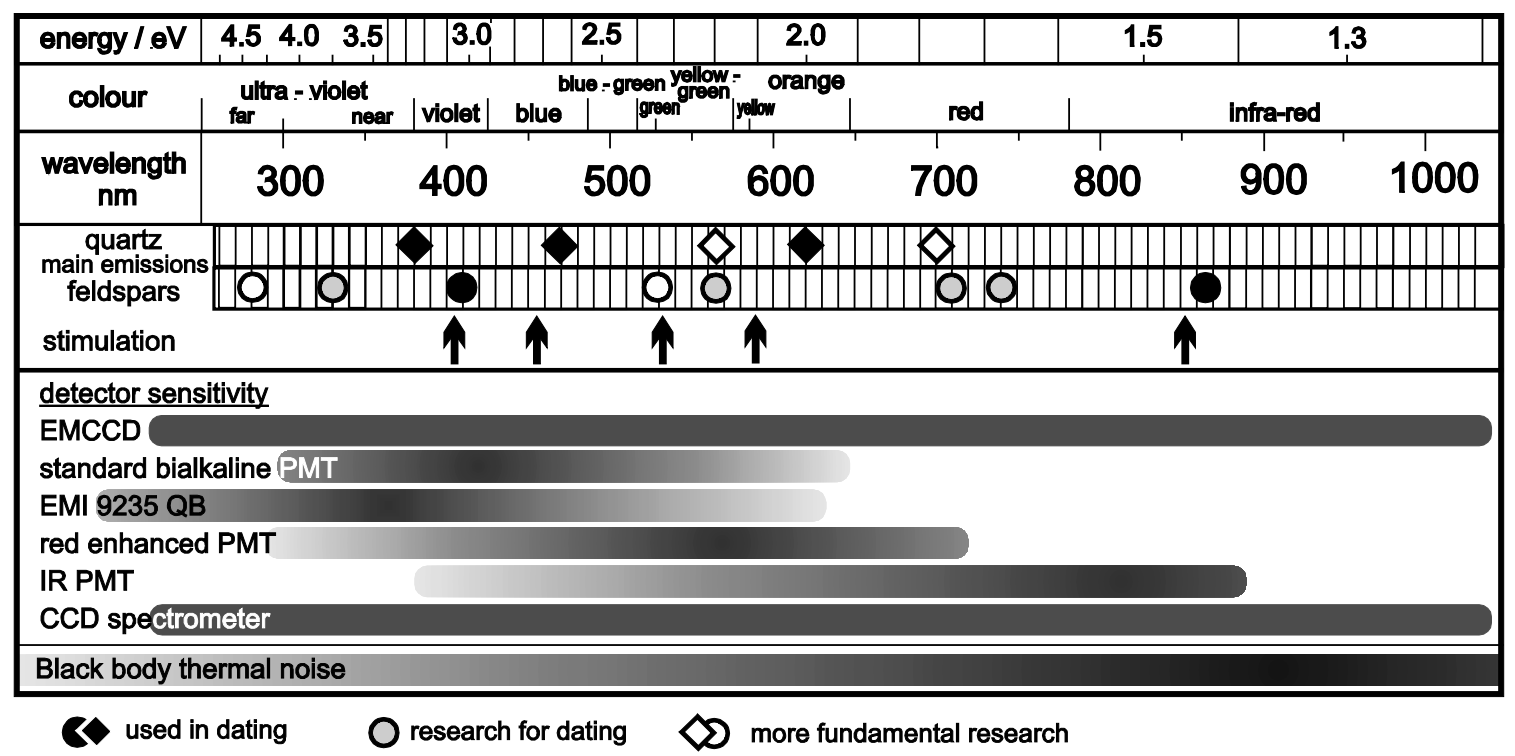

Fig. 1. Wavelengths of the main luminescence emissions of the natural minerals quartz (diamonds) and feldspar (circle) with stimulation wavelength bands of laser diodes and LEDs. The approximate sensitivity of various detectors is indicated by the intensity of the coloration over wavelength. 


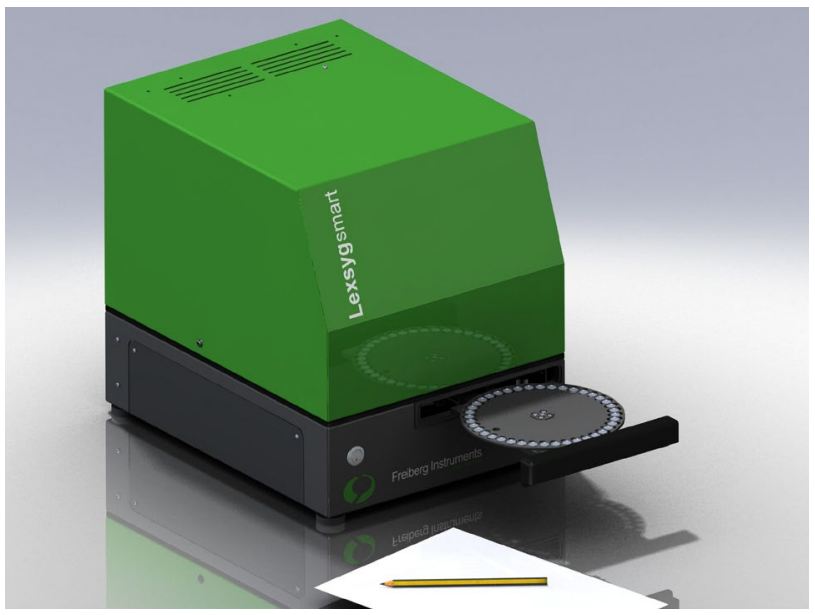

Fig. 2. The lexsyg smart luminescence system.

\section{Concept of the lexsyg smart luminescence reader}

Likewise the lexsyg research (Richter et al., 2013), the general concept of the lexsyg smart follows the separation of sample aliquot storage from the measurement chamber in order to eliminate cross bleaching effects during optical stimulation/bleaching and minimize crossirradiation (Richter et al., 2012; 2013). Due to the reduction in the distance of sample to detection, the sensitivity of the lexsyg smart is almost twice compared to the lexsyg research.

\section{Mechanical concept}

Separation is achieved by storing the aliquots awaiting measurement on a 40 position wheel for cups/discs holding the material to be measured (Fig. 3). Physical dimensions of cups/discs to be used follow the standard sizes of $10+0.0-0.2 \mathrm{~mm}$ diameter, a typical thickness of $0.5 \mathrm{~mm}$ and a total allocation of maximum height (including sample) of $2.5 \mathrm{~mm}$. The removable wheel containing the discs/cups is placed in a tray, which allows quick change of the sample wheel for efficiency. For example another wheel can be loaded during measurement allowing rapid continuation and quasi continuous use of the reader. Furthermore, it is possible to add, remove or change discs/cups during measurement when no transportation in or out of the sample wheel is taking place, which is useful in case of mistakes or the need to add additional material or aliquots and allows uninterrupted use.

The disc/cup is lifted by a piston from its position in the sample wheel and slid under the optional irradiation source or into the measurement chamber on to the heating plate (Fig. 3), similar to other approaches (e.g. Bortolot, 2000; Bortolot and Carriveau, 1982). In order to minimize gas consumption, the measurement chamber $(\sim 10 \mathrm{ml})$ is closed before any stimulation by lowering the upper part of the chamber (lid) by means of an eccentric drive mechanism, thus entirely separating the operation

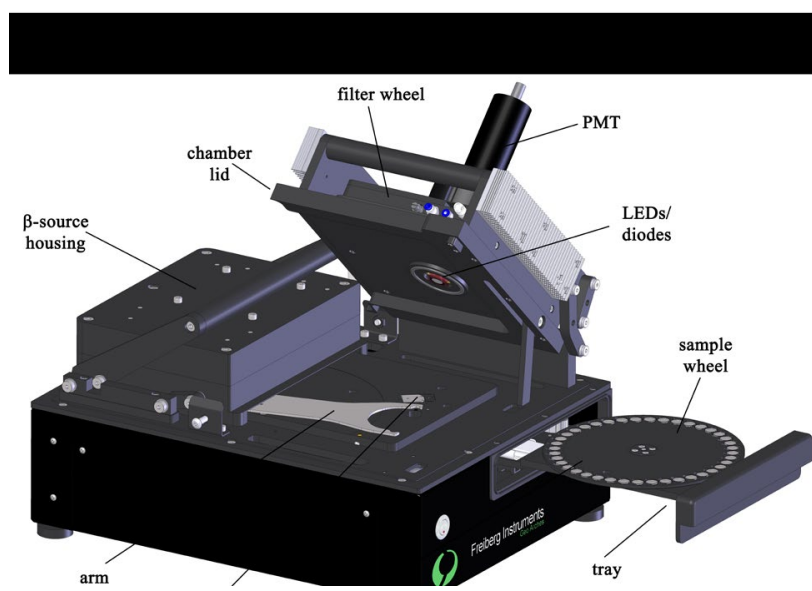

Fig. 3. The lexsyg smart system with the cover removed and chamber lid lifted for maintenance. The sample aliquots are stored on a sample wheel separate from the measurement chamber and moved to the measurement (and optional irradiation) position by an arm.

from the sample/disc reservoir (wheel). The measurement chamber containing the heating element is sealed with a quartz window (Sico SQ1) and can be evacuated as well as flooded with inert gas. With the exception of the optional filter wheel (pneumatically driven), lifting of the chamber lid and opening the source shutter, all movements are realised by electrical stepping motors.

\section{Irradiation sources}

The lexsyg smart can be optionally equipped with a single irradiation source (Fig. 3), which use is indicated by a red LED for radiation safety purposes. Due to the safe construction, shipment of the source in place is possible, and therefore, in principal, no specialized installation of the radioactive source is required.

For alpha irradiation in air a ${ }^{241} \mathrm{Am}(30 \mathrm{MBq})$ source can be used and two types of $\beta$-sources $\left({ }^{90} \mathrm{Sr}-{ }^{90} \mathrm{Y}\right)$ are available. One with an activity of $\sim 1.85 \mathrm{GBq}$ delivers approximately $0.13 \mathrm{~Gy} \mathrm{~s}^{-1}$ to coarse grain $(90-160 \mu \mathrm{m})$ quartz, while a weaker $\beta$-source $(\sim 1.6 \mathrm{GBq})$ provides a very homogeneous irradiation field (Richter et al., 2012) at the expense of a dose rate of $\sim 0.06 \mathrm{~Gy} \mathrm{~s}^{-1}$. As with the lexsyg research it possible to employ the Bremsstrahlung of the $\beta$-source for a much lower dose-rate irradiation by placing the sample for a defined time (software driven) underneath the closed source. For dosimetry purposes weaker ${ }^{90} \mathrm{Sr}^{90}{ }^{9} \mathrm{Y}$-sources delivering $\sim 0.01$ Gy s${ }^{-1}$ and $0.001 \mathrm{~Gy} \mathrm{~s}^{-1}$ can be used. Alternatively, a full housed $\mathrm{x}-$ ray irradiator based on tungsten (Varian VF-50) is available which includes a fully integrated dose rate life-time monitoring system. This equipment has the advantage of zero radiation when not in use and, depending on the chosen absorber, a useful variable dose-rate between $\sim 0.01$ and $\sim 2.1 \mathrm{~Gy} \mathrm{~s}^{-1}$ (Richter et al., in preparation). 


\section{Luminescence stimulation}

Luminescence can be stimulated in the lexsyg smart by means of heating or exposure to light. For thermal luminescence stimulation (TL) a metal plated ceramic contact heater reaching up to maximum $500^{\circ} \mathrm{C}$ (optional $710^{\circ} \mathrm{C}$ ) is implemented for homogenous sample heating with repeatability and accuracy, which has also been shown by Lomax et al. (2014). The heater can be operated at rates between 0.1 and $20.0 \mathrm{~K} \mathrm{~s}^{-1}$ with temperature calibration for each individual heater by pyrometer measurements of the surface of sample discs/cups. This allows for a precise temperature control, with the temperature lag (Kitis et al.,2015) therefore being minimized and the temperature gradient within the sample itself presumably becoming dominant. Cooling rates can be user defined and are achieved with a pump. While inert gas certainly should be used for elevated temperature procedures, the equipment can be run with compressed air as well.

Optical stimulation in the lexsyg smart can be achieved by two (optionally three) different wavelengths integrated into a stimulation unit, consisting of a total of 15 positions for LEDs or laser diodes (usually 5 for each wavelength), which are identical to the stimulation sources in the lexsyg research. Each individual LED or diode is monitored by a photodiode and a feedback loop ensures constant light power output (Fig. 4), with deviations of $<0.2 \%$, as has also been shown in Lomax et al. (2014). This ensures the same light output from all LEDs/diodes and compensates for individual differences or fluctuations, rather than from an entire array consisting of several LEDS, thus also compensating for individual aging of each LED/diode. The efficiency of the feedback loop to provide excellent reproducibility of the stimulation is also shown for a green laser source used to measure the OSL signal from $\alpha-\mathrm{Al}_{2} \mathrm{O}_{3}: \mathrm{C}$ exposed and meas-

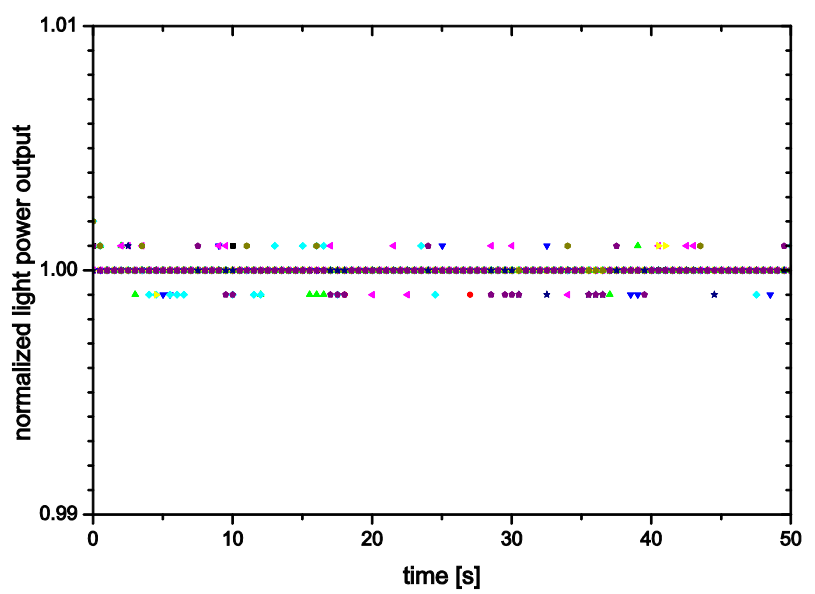

Fig. 4. Normalized light power output measured with a photodiode at $0.1 \mathrm{~mW}$ resolution for 10 individual blue LEDs $(458 \mathrm{~nm})$ set to $100 \mathrm{~mW}$. The LED output was held constant through the photodiode feedback loop via PID controller for the power regulation of each individual LED. ured to the identical dose 100 times, showing an excellent reproducibility and variation of $<1 \%$, which includes also the variation caused by the material (Fig. 5). In combination with the alignment, setup and by the use of lenses a homogeneous illumination of the area of interest $(\varnothing=6-8 \mathrm{~mm})$ by the LEDs is ensured (Fig. 6). Thus, a maximum power stability, homogeneity of stimulation light on the sample and optical power stability is provided for the stimulation wavelengths listed in Table 1. In addition each LED/diode is equipped with appropriate filters to provide, if possible, narrow stimulation wavelength bands with maximized power (Fig. 7).

These setups allow OSL measurements in continuous wave $(\mathrm{Cw}-\mathrm{OSL})$ as well as linear modulated (LM-OSL) mode at room and elevated temperatures for user specified power and duration, where the power (in $\mathrm{mW} \mathrm{cm} \mathrm{s}^{-1}$ ) at the start and at the end can be defined in LM-OSL.

\section{Luminescence detection}

It is well known that luminescence emissions at different wavelengths might correspond to different physical properties and the need for restriction of the detection wavelength window in luminescence application (Fig. 1) has been shown, e.g. by spectral analysis (e.g. Huntley et al., 1988; Krbetschek et al., 1997; Rendell et al., 1993; Townsend, 1994). The lexsyg smart can hold filters of 1 inch diameter with a total thickness up to $7.5 \mathrm{~mm}$ in a manual quick changer. However, full use of the potential of the equipment and flexibility is only achieved with an optional filter changer which is operated through the LexStudio software and allows change in detection wavelength bands during measurement sequences. This pneumatically driven filter wheel consists of 6 positions (one inch filters with a total maximum thickness of $7.5 \mathrm{~mm}$ ) each providing the possibility for changing the detection

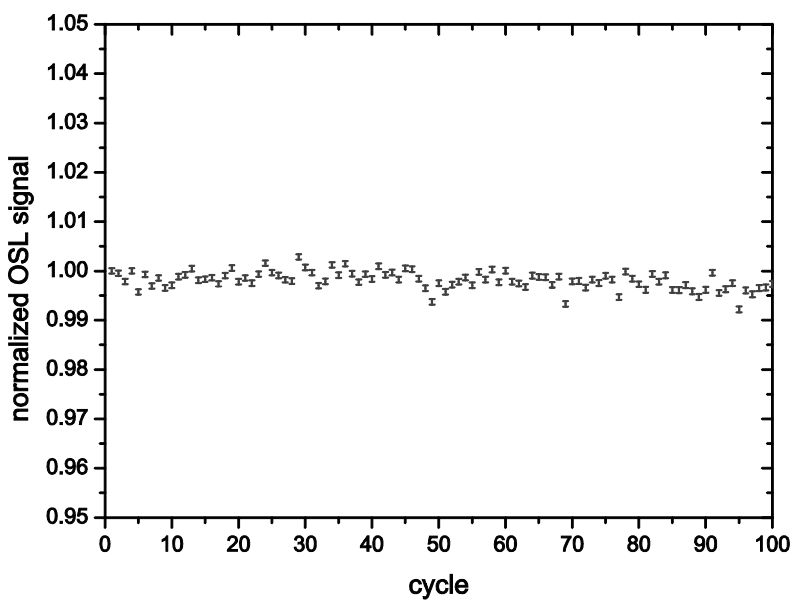

Fig. 5. First second of OSL-Signal (background 200-300 s subtracted) for a single $\alpha-\mathrm{Al}_{2} \mathrm{O}_{3}: \mathrm{C}$ crystal of 100 cycles of identical $\beta$-dose exposure, measured with a green laser and normalised to first cycle. 


\begin{tabular}{|c|c|c|c|c|}
\hline colour & type & $\begin{array}{l}\text { wavelength } \\
(\mathrm{nm})\end{array}$ & $\begin{array}{l}\sim \text { maximum power on sample } \\
\qquad\left(\mathrm{mW} \mathrm{cm}^{-2}\right)\end{array}$ & filter \\
\hline blue & LED & $458 \pm 5$ & 100 & GG420 (3 mm) + HC 458/10 \\
\hline green & LED & $525 \pm 20$ & 80 & OG515 (3 mm) + HC 530/43 \\
\hline yellow & LED & $590 \pm 30$ & 50 & BG $39(2 \mathrm{~mm})$ \\
\hline infrared & LED & $850 \pm 20$ & 300 & RG 715 (3 mm) \\
\hline violet & laser diode & $405 \pm 3$ & 100 & BG $39(1 \mathrm{~mm})+$ OD405 \\
\hline blue & laser diode & $445 \pm 3$ & 100 & GG420 (3 mm) + HC 448/20 \\
\hline infrared & laser diode & $850 \pm 3$ & 300 & RG $715(3 \mathrm{~mm})$ \\
\hline
\end{tabular}

Table 1. Colour, type and wavelength of stimulation sources with the respective delivered power (maximum limited by software and calculated for the central $8 \mathrm{~mm}$ diameter area) on the sample position after passing through the filters. Standard sets are marked by a grey background.

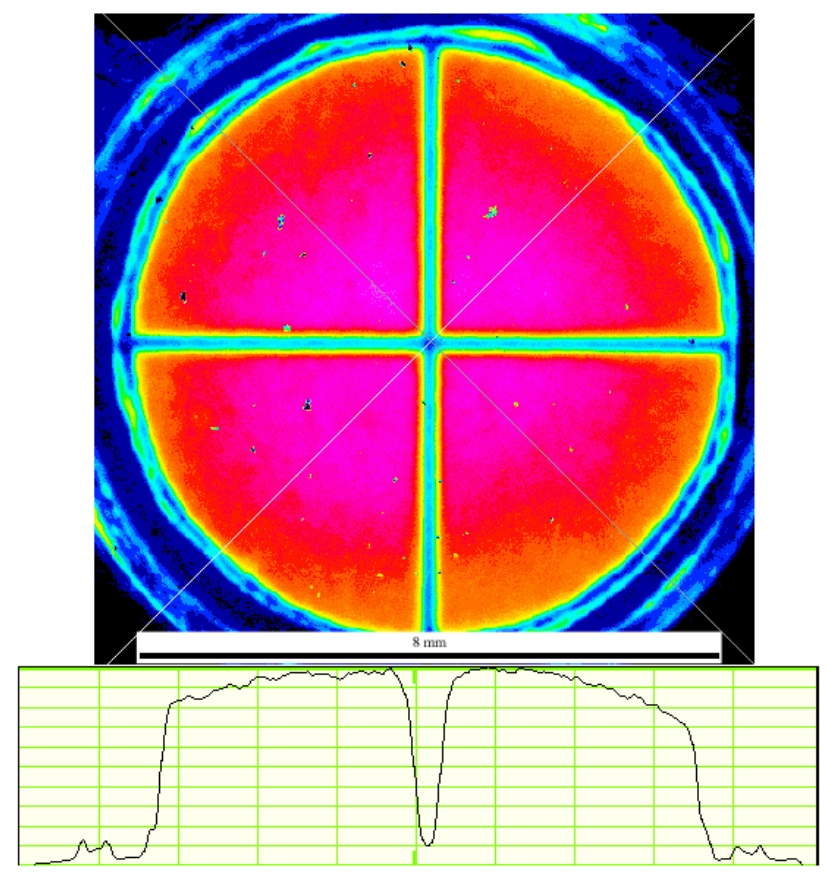

Fig. 6. Relative reflected light measurement showing the homogeneity of illumination by IR-LEDs $(850 \pm 20 \mathrm{~nm})$ on a sample cup lined with white paper. Note the homogeneity over the central area of interest and the reduced reflectance in the form of a cross in the centre caused by reference lines.

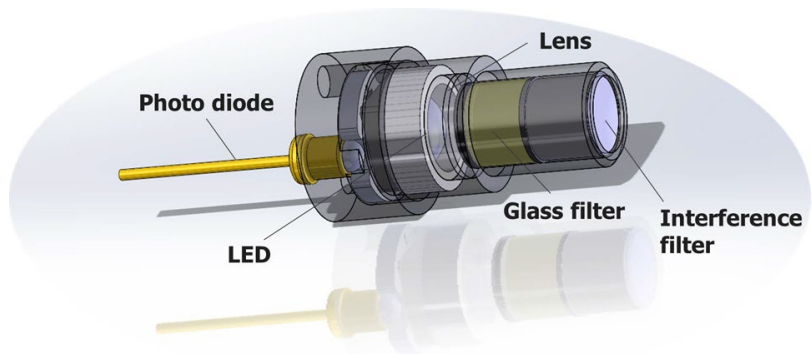

Fig. 7. Schematic drawing of the setup of an individual LED with lens, filters and the photodiode for the feedback loop to the PID which controls the power for the LED to provide stability.

window between measurements within 0.3 to $3.0 \mathrm{~s}$. Table 2 lists standard sets of filters for luminescence application with some selected materials. Filter combinations were optimized for best blocking of stimulation light (OSL) and/or thermal noise (TL, black-body radiation), while still providing maximum transmission in the desired wavelength range.

The lexsyg smart can be equipped with a single detector. For most applications standard PMTs are sufficient and the choice depends largely on the detection window and desired efficiency. Most commonly the bi-alkaline photomultiplier tube from Hamamatsu (H7360-02) with a range from $300-650 \mathrm{~nm}$ (27\% quantum efficiency at $400 \mathrm{~nm}$ ) is suitable, while an extension into UV detection is achievable with the EMI (9235QB), providing a detec-

\begin{tabular}{|c|c|c|c|}
\hline use & filter combination & stimulation & material \\
\hline Violet OSL - $340 \mathrm{~nm}$ & U340 + HC 340/26 & Laser diodes (405 nm) & quartz \\
\hline Blue OSL - 340 nm & $\mathrm{U} 340+\mathrm{HC} 340 / 26$ & LED $(458 \mathrm{~nm})$ & quartz, $\mathrm{Al}_{2} \mathrm{O}_{3}, \mathrm{BeO}$ \\
\hline Blue OSL - $365 \mathrm{~nm}$ & U340 + BP $365 / 50$ & LED (458 nm) & quartz, $\mathrm{Al}_{2} \mathrm{O}_{3}, \mathrm{BeO}$ \\
\hline Green OSL - 365 nm & BG $3+$ BP $365 / 50$ & LED (525 nm) & quartz, $\mathrm{Al}_{2} \mathrm{O}_{3}$ \\
\hline Yellow OSL - $330 \mathrm{~nm}$ & $\mathrm{BG} 3+\mathrm{HC} 340 / 26$ & LED $(590 \mathrm{~nm})$ & quartz, $\mathrm{Al}_{2} \mathrm{O}_{3}$ \\
\hline Yellow OSL - $365 \mathrm{~nm}$ & BG $3+$ BP $365 / 50$ & LED (590 nm) & quartz, $\mathrm{Al}_{2} \mathrm{O}_{3}$ \\
\hline Yellow OSL - $410 \mathrm{~nm}$ & $\mathrm{BG} 3+\mathrm{HC} 414 / 46$ & LED $(590 \mathrm{~nm})$ & feldspar, $\mathrm{Al}_{2} \mathrm{O}_{3}$ \\
\hline IRSL - $410 \mathrm{~nm}$ & BG $39+$ HC 414/46 & LED / Laser diodes (850 nm) & feldspar \\
\hline IRSL - $565 \mathrm{~nm}$ & BG $39+$ HC 575/25 & LED / Laser diodes (850 nm) & feldspar \\
\hline IRSL wideband blue & $B G 39+B G 25+K G 3$ & LED / Laser diodes (850 nm) & feldspar \\
\hline TL wideband blue & $B G 39+B G 25+K G 3$ & heating element & flint, quartz, LiF \\
\hline $\mathrm{TL}-330 \mathrm{~nm}$ & $\mathrm{U} 340$ + HC 340/26 & heating element & quartz \\
\hline $\mathrm{TL}-380 \mathrm{~nm}$ & $\mathrm{BG} 3+\mathrm{BP} 365 / 50$ & heating element & quartz \\
\hline $\mathrm{TL}-410 \mathrm{~nm}$ & BG $39+\mathrm{HC} 414 / 46$ & heating element & feldspar \\
\hline $\mathrm{TL}-565 \mathrm{~nm}$ & BG $39+$ HC $575 / 25$ & heating element & feldspar, quartz \\
\hline
\end{tabular}

Table 2. Suggested filter sets for some selected materials with standard sets on a grey background. 
tion range between 160 and $630 \mathrm{~nm}$ with $30 \%$ quantum efficiency. The beneficial use of a red-enhanced UV-VISPMT for more efficient detection of luminescence emission at e.g. $620 \mathrm{~nm}$ for TL of quartz has been shown in a recent study (Richter et al., 2015), employing the Hamamatsu H7421-40 thermoelectric/air cooled GaAsP photocathode PMT with a sensitivity between 300 and $720 \mathrm{~nm}$ and a quantum efficiency of $40 \%$ at $580 \mathrm{~nm}$. Infrared luminescence detection can be achieved with a thermoelectric/air cooled $\left(-10^{\circ} \mathrm{C}\right) \mathrm{GaAs}$ photocathode PMT Hamamatsu H7421-50, providing a general sensitivity between 380 and $890 \mathrm{~nm}$, with a peak of $12 \%$ quantum efficiency at $800 \mathrm{~nm}$. In principle, any detection system can be mounted on a lexsyg smart.

\section{SOFTWARE LEXSTUDIO AND LEXEVA}

The software LexStudio for operating the lexsyg smart allows easy programming of individual as well as standard measurement sequences. The graphical user interface combines clear arrangement with usability and provides sequence editing, device controlling and live viewing of measurements, within a user account management system. Parameter selection ranges are software restricted for safe operation of the lexsyg smart, while pre-sets can be defined by the user. Such are notably the choice of detection window for the selected stimulation type/wavelength in order to prevent overexposure of the detection system. Heating rates, stage duration times, maximums temperatures as well as cooling temperatures, whether for preheats, TL or elevated temperature meas- urements can be set. Channel integration times, delayed stimulation or detection start times as well as their durations can be changed or predefined.

The generation of cyclic measurements, e.g. SARprotocol (Murray and Wintle, 2000) can be tedious and is prone to error especially for novices. A SAR Plug-in (SARPI) helps with the quick generation of standard SAR sequences which are based on the user defined pre-sets of an approximation of the expected palaeodose, test-dose, preheat/cutheat temperature, read temperature and stimulation time (Fig. 8). Other user defined parameters, which are less frequently changed, like preheat/cutheat durations, stimulation powers, heating rates, channel width, cooling temperatures, etc. can be set in a parameter table (advanced options) depending on user authorization level. The irradiation times for regeneration dose points are express as percentage of the expected palaeodose and the number of regeneration dose points can be freely set, while including an obligatory recycling point. Optionally, an IR-depletion test can be automatically implemented for the last dose point to be measured and the software provides feedback if IR-depletion and recuperation points were omitted. To further aid in SAR sequence generation the general setup of measurements is visualized as a graph showing the dose points set on a theoretical dose response curve according to a user set simple mathematical model and estimated $\mathrm{D}_{0}$. The various dose points are colour coded for visual verification of the principal correctness of the sequence (Fig. 8). Obviously choices for the stimulation wavelength and the detection window have to be made as well. The SARPI feature then auto-

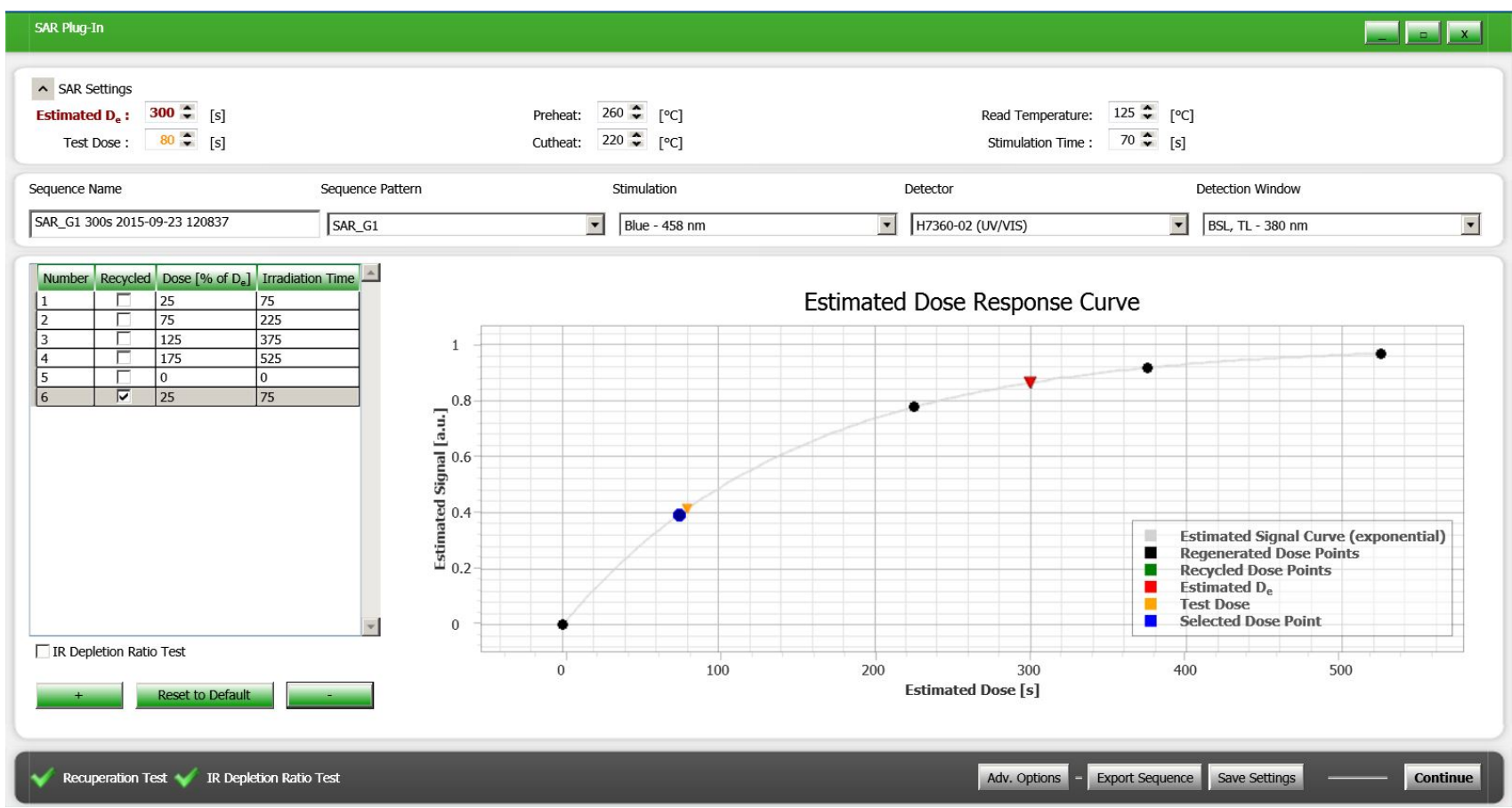

Fig. 8. Screenshot of SARPI, a LexStudio software plugin for the simple generation of standard SAR sequences for lexsyg devises. 
matically generates a SAR cycle according the user defined parameters. The resulting SAR sequence based on such a rather standardized approach is often appropriate, but it can be modified like any other measurement sequence according further user needs.

The acquired data are stored together with the measurement parameters in a specific lexsyg format (xsyg), which includes not only the luminescence counts but also e.g. stimulation power over time, temperature during OSL measurements, etc. By providing such a full data set it is possibly to analyse and check the entire measurement and its parameters, as is required for physical measurements. Additional export for further data treatment is possible for ASCII and the luminescence data can be also recorded as bin/binx-format for processing in Analyst (Duller, 2015). The evaluation software LexEva provides the link to the R-package 'luminescence' (Kreutzer et al., 2012) for data evaluation in luminescence dating application, while many other operations can be performed within LexEva (LexEva - User manual). For the analysis of artificial or other dosemeters the software TLStudio (TLStudio - User manual). is available, which was designed especially for TL-dosimetry and also reads and analyses the lexsyg generated files.

\section{CONCLUSIONS}

The lexsyg smart system is a versatile tool for standard mass luminescence measurements, while providing flexibility for the adaptation of measurements protocols by user interference to many measurement parameters like heating rates, maximum temperature, stimulation power and duration. It therefore also serves research in luminescence dosimetry as well as dating application. Cross-irradiation and -talk is minimized by the separation of measurement chamber and sample storage. It can be used for most dosimetric materials because it provides several stimulation possibilities. The lexsyg smart is capable of the excitation of luminescence by thermal, as well as violet, blue, green, yellow or IR stimulation, which can be measured by a single detector. Detection wavelength bands can be automatically changed with a filter wheel holding 6 slots to restrict luminescence detection to the appropriate wavelength band. Samples can be irradiated either by $\alpha$ - or $\beta$-sources, and an x-ray generator can provide a flexible range of dose-rates.

\section{ACKNOWLEDGEMENTS}

We would like to thank the entire engineering and scientific team at Freiberg Instruments. Such developments are only achievable with the input, feedback and advice of many scientists and we're indebted to all colleagues who have been involved. We gratefully acknowledge their help in this achievement and their testing of the instrument. These contributions played an important role and made this development possible.

\section{REFERENCES}

Aitken MJ, 1985. Thermoluminescence Dating. London, Academic Press: 359pp.

Aitken MJ, 1998. An Introduction to Optical Dating. The Dating of Quaternary Sediments by the Use of Photon-stimulated Luminescence. Oxford University Press, Oxford: 280pp.

Bortolot VJ, 2000. A new modular high capacity OSL reader system. Radiation Measurements 32(5-6): 751-757, DOI 10.1016/S13504487(00)00038-X.

Bortolot VJ and Carriveau GW, 1982. An integrated TL measurement and computer system. PACT, Revue du groupe européen d'études pour les techniques physiques, chimiques et mathématiques appliquées à l'archéologie 6: 272-281.

Bøtter-Jensen L and Bundgaard J, 1978. An automated reader for thermoluminescent dating. PACT, Revue du groupe européen d'études pour les techniques physiques, chimiques et mathématiques appliquées à l'archéologie 2: 48-57.

Bøtter-Jensen L, McKeever SWS and Wintle AG, 2003. Optically Stimulated Luminescence Dosimetry. Amsterdam, Elsevier, 374pp.

Duller GAT, 2015. The Analyst software package for luminescence data: overview and recent improvements. Ancient TL 33(1): 35-42.

Guérin G and Lefèvre J-C, 2014. A low cost TL-OSL reader dedicated to high temperature studies. Measurement 49: 26-33, DOI: 10.1016/j.measurement.2013.11.035.

Huntley DJ, Godfrey-Smith DI, Thewalt MLW and Berger GW, 1988. Thermoluminescence spectra of some mineral samples relevant to thermoluminescence dating. Journal of Luminescence 39: 123136, DOI 10.1016/0022-2313(88)90067-1.

Kitis G, Kiyak NG an Polymeris GS, 2015. Temperature lags of luminescence measurements in a commercial luminescence reader. $\mathrm{Nu}$ clear Instruments and Methods in Physics Research Section B. Beam Interactions with Materials and Atoms 359: 60-63, DOI 10.1016/j.nimb.2015.07.041.

Kreutzer S, Schmidt C, Fuchs MC, Dietze M, Fischer M and Fuchs M, 2012. Introducing an $\mathrm{R}$ package for luminescence dating analysis. Ancient TL 30(1): 1-8.

Krbetschek MR, Götze J, Dietrich A and Trautmann T, 1997. Spectral information from minerals relevant for luminescence dating. Radiation Measurements 27(5-6): 695-748, DOI 10.1016/S13504487(97)00223-0.

LexEva - Manual for luminescence data analysis acquired with lexsyg devices.

Lapp T, Jain M, Thomsen KJ, Murray AS and Buylaert J-P, 2012. New luminescence measurement facilities in retrospective dosimetry. Radiation Measurements 47(9): 803-808, DOI 10.1016/j.radmeas.2012.02.006.

Lapp T, Kook M, Murray AS, Thomsen KJ, Buylaert JP and Jain M, 2015. A new luminescence detection and stimulation head for the Risø TL/OSL reader. Radiation Measurements 81: 178-184, DOI: 10.1016/j.radmeas.2015.02.001.

Lomax J, Kreutzer S and Fuchs M, 2014. Performance tests using the Lexsyg luminescence reader. Geochronometria 41(4): 327-333, DOI 10.2478/s13386-013-0174-x.

McKeever SWS, 1985. Thermoluminescence of solids. Cambridge, Cambridge University Press, 392.

Mejdahl V, 1982. An automated procedure for the thermoluminescence dating of pottery and burnt stones. PACT, Revue du groupe européen d'études pour les techniques physiques, chimiques et mathématiques appliquées à l'archéologie 7: 83-96.

Murray AS and Wintle AG, 2000. Luminescence dating of quartz using an improved single-aliquot regenerative-dose protocol. Radiation Measurements 32(1): 57-73, DOI 10.1016/S1350-4487(99)00253$\mathrm{X}$.

Rendell HM, Khanlary MR, Townsend PD, Calderón T and Luff BJ, 1993. Thermoluminescence spectra of minerals. Mineralogical Magazine 57: 217-222.

Richter D, Pintaske R, K.Dornich and Krbetschek M, 2012. A novel beta source design for uniform irradiation in dosimetric applications. Ancient TL 30(2): 57-63. 


\section{Richter et al.}

Richter D, Richter A and Dornich K, 2013. lexsyg - a new system for luminescence research. Geochronometria 40: 220-228, DOI 10.2478/s13386-013-0110-0.

Richter D, Mittelstrass D, Kreutzer S, Fuchs M and Dornich K, in preparation. An X-ray irradiator for dosimetric application.

Richter D, Klinger P and Zöller L, 2015. Palaeodose underestimation of heated quartz in red-TL dating of volcanic contexts. Geochrono- metria 42: 182-188, DOI 10.1515/geochr-2015-0020.

TLStudio - User manual for TLD-analysis.

Townsend PD, 1994. Analysis of TL emission spectra. Radiation Measurements 23(2-3): 341-348, DOI 10.1016/1350-4487(94)90062-0.

Yukihara EG and McKeever SWS, 2011. Optically stimulated luminescence: Fundamentals and applications. Oxford, Wiley-Blackwell, 388pp. 